ISSN 1112-9867

http://www.jfas.info

\title{
MORTAR INCORPORATING SUPPLEMENTARY CEMENTITIOUS MATERIALS: STRENGTH, ISOTHERMAL CALORIMETRY AND ACIDS ATTACK
}

\author{
Y. Senhadji ${ }^{1,2^{*}}$, A.S. Benosman ${ }^{2}$, G. Escadeillas ${ }^{3}$, M. Mouli ${ }^{2}$, L. Laoufi ${ }^{1}$ and H. Khelafi ${ }^{2}$ \\ ${ }^{1}$ Département de génie civil, Université de Mascara, Mascara 29000, Algérie. \\ ${ }^{2}$ Département de génie civil, Laboratoire Lab-Mat, ENP-Oran El Mnaouer 31000, Algérie. \\ ${ }^{3}$ Département de génie civil, Lab. LMDC, Université Paul Sabatier, Toulouse, France.
}

Received: 08 Nouvembre 2015 / Accepted: 20 Mars 2016 / Published online: 01 May 2016

\begin{abstract}
Supplementary cementitious materials (SCMs) prove to be effective to meet most of the requirements of durable concrete and leads to a significant reduction in $\mathrm{CO}_{2}$ emissions. This research studies the effect different SCMs (natural pozzolan (PN) / limestone fine (FC) at various replacement levels) on the physical and mechano-chemical resistance of blended mortar. The paper primarily deals with the characteristics of these materials, including heat of hydration, strength and effects of aggressive chemical environments (using sulphuric acid and nitric acid). Over 6 mixes were made and compared to the control mix. Tests were conducted at different ages up to 360 days. The experimental results in general showed that Algerian mineral admixtures (PN/FC) were less vulnerable to nitric and sulphuric acid attack and improved the properties of mortars, but at different rates depending on the quantity of binder.
\end{abstract}

Keywords: natural pozzolan; portlandite; limestone fine; durability; blended cements.

Author Correspondence, e-mail: senhadjidz@yahoo.fr

doi: http://dx.doi.org/10.4314/jfas.v8i2.4 


\section{INTRODUCTION}

La fabrication du ciment s'accompagne d'un dégagement important de $\mathrm{CO}_{2}$. L'industrie cimentière algérienne a déjà diminué ces émissions en améliorant le rendement énergétique de ses fours ; en revanche, la part «chimique»des émissions, due à la décarbonatation du calcaire, est pratiquement irréductible [1].

La valorisation des matériaux locaux, peu ou pas exploités, dans la construction est devenue actuellement une solution nécessaire aux problèmes économiques des pays, notamment des pays en voie de développement. C'est dans ce contexte que s'ouvre la réflexion sur la recherche de nouveaux bétons capables de résoudre les problèmes économiques et techniques, rencontrés dans le domaine de la construction. Parmi ces matériaux, on trouve les ajouts de remplacement d'origine minérale qui sont largement exploités pour la fabrication des ciments aux additions minérales dans le monde. Du point de vue économique, ils représentent un facteur très important dans la production du ciment avec ajout minéral (C.P.J-C.E.M II), du moment que la consommation en clinker baisse en fonction du taux d'ajout utilisé. La réduction de la proportion du clinker dans la fabrication du ciment avec ajout minérale présente les avantages suivants :

- réduire la consommation des ressources locales tels que les gisements de calcaire ;

- réduction de la consommation d'énergie,

- réduction des émissions de $\mathrm{CO}_{2}$ (provenant de la décarbonatation du calcaire et la combustion lors de la cuisson) [2-4].

Les pouzzolanes sont des matériaux naturels ou artificiels riches en silice et en alumine capables de réagir avec la chaux en présence de l'eau et de former à l'issue de cette réaction des produits manifestant des propriétés liantes. La chaux libérée au cours de l'hydratation des composés du clinker réagit avec le matériau pouzzolanique à l'intérieur du matériau cimentaire pour former des nouveaux composés qui participent aux résistances mécaniques du mortier ou du béton.

Cette alternative ne suscite encore qu'un intérêt limité de la part des professionnels du béton prêt à l'emploi et de la préfabrication, confrontés aux limites de la réglementation et à l'absence d'une étude nationale approfondie sur le sujet. 
Le travail proposé ici vise à étudier l'impact des additions minérales locales sur les propriétés de calorimétrie semi-adiabatique, les caractéristiques mécaniques des différents liants ainsi que sur leur durabilité.

\section{MATERIAUX ET PROCEDURES EXPERIMENTALES}

\subsection{Matériaux}

Ciment: Le ciment utilisé dans la confection des eprouvettes de mortier est un ciment type CEM I 42,5, fabriqué par la cimenterie de Zahana (Algérie). Sa masse volumique absolue est de $3100 \mathrm{~kg} / \mathrm{m}^{3}$ et sa surface spécifique de $325 \mathrm{~m}^{2} / \mathrm{kg}$. La plupart des essais de caractérisation physiques et chimiques ont été réalisés conformément aux normes AFNOR. Les caractéristiques chimiques et minéralogiques sont présentées dans le tableau 1.

Tableau 1. Composition chimique et minéralogique du ciment (CEMI) et des ajouts minéraux

\begin{tabular}{|c|c|c|c|c|c|c|c|c|c|c|c|}
\hline \multirow{2}{*}{$\begin{array}{l}\text { Type du } \\
\text { matériau }\end{array}$} & \multirow[t]{2}{*}{ Densité } & \multirow{2}{*}{$\begin{array}{l}\text { Finesse } \\
\left(\mathrm{m}^{2} / \mathrm{kg}\right)\end{array}$} & \multicolumn{9}{|c|}{ Composition chimique (\%) } \\
\hline & & & $\mathrm{SiO}_{2}$ & $\mathrm{CaO}$ & $\mathrm{Al}_{2} \mathrm{O}_{3}$ & $\mathrm{Fe}_{2} \mathrm{O}_{3}$ & $\mathrm{MgO}$ & $\mathrm{K}_{2} \mathrm{O}$ & $\mathrm{SO}_{3}$ & $\mathrm{Na}_{2} \mathrm{O}$ & $\mathrm{CaCO}_{3}$ \\
\hline Clinker & 3.10 & 325 & 21.4 & 63.89 & 4.59 & 5.52 & 1.37 & 0.41 & 2.72 & 0.13 & -- \\
\hline $\mathrm{PN}^{*}$ & 2.62 & 430 & 47.2 & 9.99 & 18.85 & 10.84 & 4.38 & 0.50 & 0.20 & 0.81 & -- \\
\hline $\mathrm{FC}^{* *}$ & 2.70 & 356 & 7.89 & 45.45 & 2.58 & 1.13 & 1.72 & 0.95 & 0.21 & 0.00 & 86.98 \\
\hline \multicolumn{3}{|c|}{$\mathbf{C}_{2} \mathbf{S}=25.69 \%$} & \multicolumn{3}{|c|}{$\mathbf{C}_{3} \mathbf{S}=47.15 \%$} & \multicolumn{3}{|c|}{$\mathbf{C}_{3} \mathbf{A}=2.84 \%$} & \multicolumn{3}{|c|}{$\mathbf{C}_{4} \mathbf{A F}=16.70 \%$} \\
\hline
\end{tabular}

*: pouzzolane naturelle et $* *$ : fillers calcaires

Additions: Deux types d'additions, qui sont en abondance dans l'ouest algérien à cause de leur nature géologique, ont été utilisés dans cette étude : la pouzzolane naturelle (PN) et les fillers calcaires (FC).

- La pouzzolane naturelle: La PN utilisée est de provenance du gisement de Bouhamidi à Béni-Saf (Algérie), extraite à la côte $210 \mathrm{~m}$. Elle fut approvisionnée par l'entreprise SPMC de Beni-Saf (groupe FERPHOS) en quantité suffisante pour nos besoins à l'élaboration du plan expérimental. Toute la PN utilisée comme substituant au ciment est homogénéisée, séchée, concassée et réduite en poudre $(80 \mu \mathrm{m})$ à l'aide d'un micro broyeur.

- Les Fillers calcaires: Les FC sont obtenu par broyage poussé des déchets de concassage provenant de la carrière de Kristel située à Oran (Algérie). Les caractéristiques physiques ainsi que la composition chimique des FC sont présentées dans le tableau 1. Les FC sont constitués essentiellement de calcite $\left(\mathrm{CaCO}_{3}\right)$. 
La détermination des caractéristiques physiques et chimiques de la PN et des FC a été réalisée en collaboration avec le laboratoire de la cimenterie de Zahana. Les résultats de ces analyses sont reportés dans le tableau 1 et les résultats de la diffraction aux rayons $\mathrm{X}$ sont montrés par la figure 1 et la figure 2 pour la $\mathrm{PN}$ et le FC respectivement.

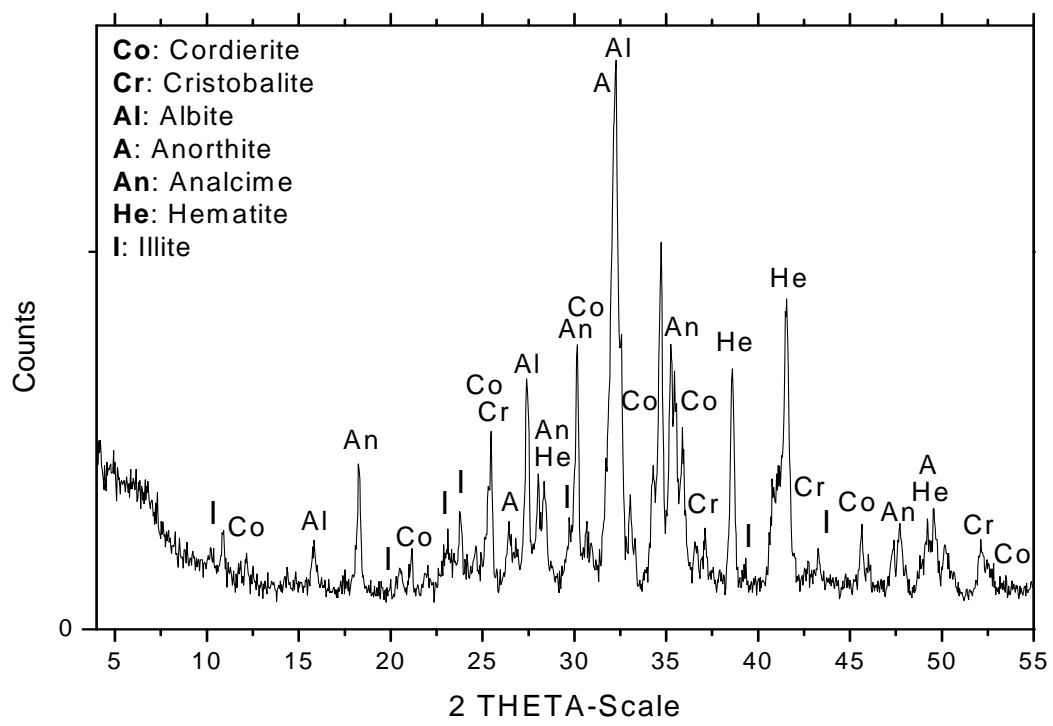

Fig.1. Diffractogramme de rayons $\mathrm{X}$ de la pouzzolane naturelle

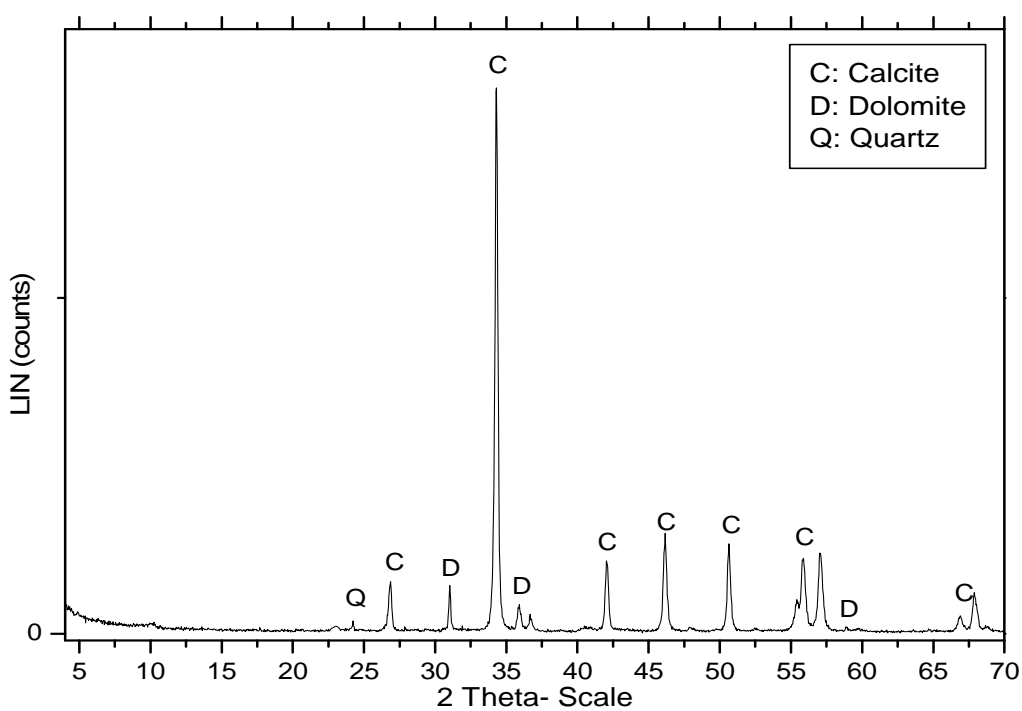

Fig.2. Diffractogramme de rayons $\mathrm{X}$ des fillers calcaire 
Le sable: C'est un sable de nature calcaire concassé obtenu de dimension 0/3 mm. Afin d'avoir un sable dont la courbe granulométrique se situe à l'intérieur du fuseau normalisé, nous avons procédé à une correction de la granulométrie.

\subsection{La calorimétrie}

La chaleur dégagée lors de l'hydratation des ciments est suivie par calorimétrie semi-adiabatique de Langavant selon la norme européenne NF EN 196-9 [5] en mesurant à intervalles de temps réguliers la température au cœur de mortiers. Le mélange de mortier standard se compose de $1080 \mathrm{~g}$ de sable, $144 \mathrm{~g}$ d'eau et $360 \mathrm{~g}$ de liant. Nous avons choisi d'étudier le mortier témoin ainsi que les mortiers PN20 et FC10. A une échéance donnée, la chaleur d'hydratation du ciment contenu dans l'éprouvette est égale à la somme de la chaleur accumulée dans le vase et de la chaleur dissipée vers le milieu ambiant depuis l'instant initial.

\subsection{Confection des éprouvettes et condition de conservation}

Le mortier utilisé dans notre étude, se compose de la proportion en masse suivante : (1 ciment, 3 sable et un rapport eau sur ciment $\mathrm{E} / \mathrm{C}=0.6$ constant pour tous les mortiers). La valeur du rapport E/C à été choisie pour obtenir un mortier maniable, selon la norme ASTM C 1437.

Deux types des éprouvettes de mortiers sont confectionnés selon la norme NF EN 196-1[6]. Dans le premier type d'éprouvettes nous avons incorporé de la PN en remplacement par masse du ciment à différents dosages $(0,15,20$ et $25 \%)$.

Dans le deuxième type d'éprouvettes, les FC ont été incorporés avec les dosages de $(5,10$ et 15\%). La substitution est faite par rapport à la masse du ciment CEMI 42,5 de Zahana. Pour la caractérisation de la résistance mécanique les éprouvettes sont de dimension (40 x 40 x 160) $\mathrm{mm}^{3}$ et de dimension $(50 \times 50 \times 50) \mathrm{mm}^{3}$ pour la résistance chimique.

Après démoulage à $24 \mathrm{~h}$ et une cure sous l'eau de 28 jours. Les premiers $\left(40\right.$ x 40 x 160) $\mathrm{mm}^{3}$ ont subi des essais de traction par flexion puis les demi-prismes obtenus ont été rompus en compression à différents âges $(7,28$ et 90j). Les éprouvettes sont conservées dans l'eau saturée en chaux $(3 \mathrm{~g} / 1 \mathrm{de} \mathrm{CaO})$ à $20{ }^{\circ} \mathrm{C}$ jusqu'aux échéances de rupture. Les deuxièmes $50 \times 50 \times 50 \mathrm{~mm}^{3}$ sont mises en solution acide de même concentration $5 \% \mathrm{HNO}_{3}$ et $5 \% \mathrm{H}_{2} \mathrm{SO}_{4}$. Des essais de mesure de masse sont effectués jusqu'au $56^{\text {ème }}$ jour selon la norme ASTM C267-96 [7]. Les sept séries de mortier ainsi obtenus sont désignés respectivement par : 
CEM I (mortier témoin sans addition), PN15 (15\% de PN), PN20 (20\% de PN), PN25 (25\% de PN), FC5 (5\% de FC), FC10 (10\% de FC), FC15 (15\% de FC).

\section{RESULTATS ET INTERPRETATIONS}

\subsection{Chaleur d'hydratation}

Le résultat de la chaleur d'hydratation et du flux de chaleur des mortiers choisis (CEM I, NP20 et FC10) est représenté sur les figures 3 et 4. Les valeurs des flux maximum ainsi que la chaleur d'hydratation à la fin de l'essai sont reprsentées dans le tableau 2. Nous constatons que la chaleur d'hydratation est quasiment stabilisée au bout de 5 jours, ceci indique que la réaction ne produit pas de dégagement de chaleur supplémentaire.

Tableau 2. Caractéristiques d'hydratation des différents liants

\begin{tabular}{ccccc}
\hline Mortier & $\begin{array}{c}\text { \% de l'ajout } \\
\text { minéral }\end{array}$ & $\begin{array}{c}\mathbf{T}{ }^{\circ} \mathbf{C} \\
\text { maximale }\end{array}$ & $\begin{array}{c}\text { La chaleur d'hydratation } \\
\mathbf{1 2 0} \mathbf{~ h}(\mathbf{J} / \mathbf{g})\end{array}$ & $\begin{array}{c}\text { Flux de chaleur } \\
\text { Max. }(\mathbf{J} / \mathbf{g} / \mathbf{h})\end{array}$ \\
\hline CEM I & 0 & 59,4 & 441,5 & 65,40 \\
PN20 & 20 & 51,6 & 325,1 & 46,75 \\
FC10 & 10 & 50,1 & 374,9 & 32,86 \\
\hline
\end{tabular}

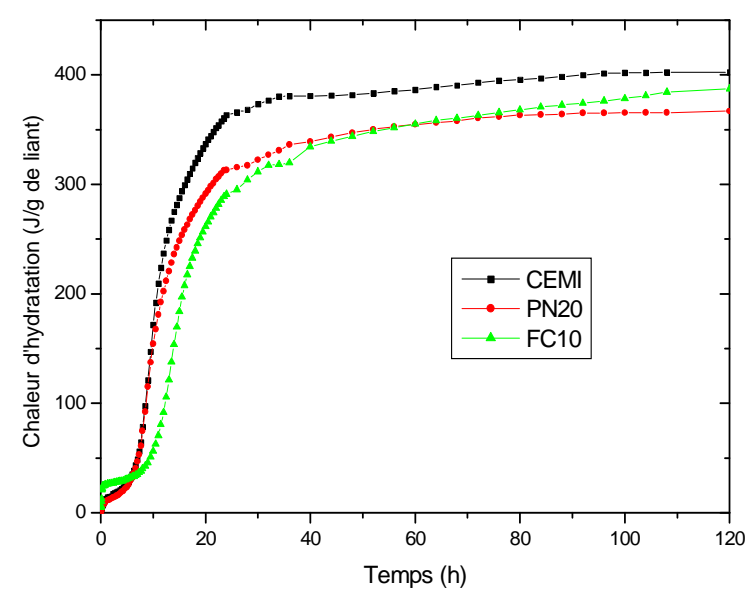

Fig.3. Evolution de la chaleur d'hydratation

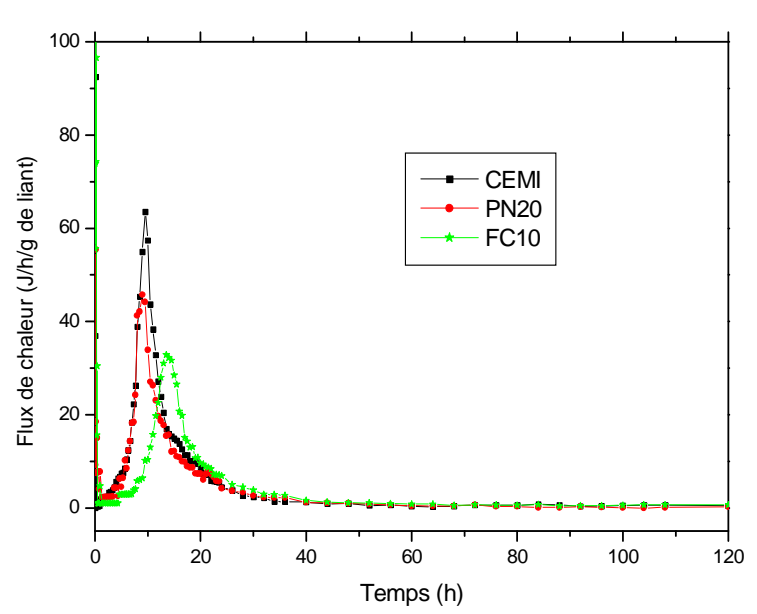

Fig.4. Evolution du flux de chaleur

Pour un rapport E/L constant, la chaleur d'hydratation ainsi que le flux de chaleur maximum diminuent avec la substitution de la PN au CEM I. Le même constat peut être établi pour le 
mortier à base de FC. L'hydratation du ciment est très affectée par la température, lorsque la température est plus élevée l'hydratation se produit plus rapidement. La quantité de chaleur dégagée pendant l'hydratation dépend de la quantité et du type de ciment dans le mortier.

La chaleur dégagée par le CEM I est plus importante que les chaleurs dégagées par les mortiers PN20 et FC10. Ceci s'explique par le fait que la proportion de ciment contenue dans le CEM I est plus importante. Le remplacement de 20 et $10 \%$ du ciment par la PN et des FC respectivement, diminue le second pic du flux d'hydratation de 26 et $15 \%$ respectivement. La période d'atteindre le taux maximum d'évolution de la chaleur a été retardée seulement pour le mélange avec des FC.

\subsection{Les résistances mécaniques}

Le tableau 3 présente les résultats de la résistance à la flexion et de compression des différents mortiers à différents âges.

Tableau 3. Résultats de la résistance à la flexion et la résistance à la compression

\begin{tabular}{ccccccc}
\hline \multirow{2}{*}{$\begin{array}{c}\text { Mortier } \\
\text { (jours) }\end{array}$} & \multicolumn{3}{c}{ Resistance à la flexion } & \multicolumn{3}{c}{ Resistance à la compression } \\
\cline { 2 - 7 } & $\mathbf{7}$ & $\mathbf{2 8}$ & $\mathbf{9 0}$ & $\mathbf{7}$ & $\mathbf{2 8}$ & $\mathbf{9 0}$ \\
\hline CEM I & 7.66 & 7.79 & 8.21 & 32.3 & 45.5 & 58.8 \\
\hline PN15 & 6.97 & 7.07 & 8.46 & 27.4 & 39.6 & 53.4 \\
\hline PN20 & 5.47 & 6.50 & 7.02 & 27.9 & 41.8 & 52.5 \\
\hline PN25 & 4.62 & 5.34 & 6.82 & 27.1 & 37.8 & 48.6 \\
\hline FC5 & 7.60 & 7.63 & 8.58 & 37.1 & 38.9 & 42.6 \\
\hline FC10 & 6.92 & 7.36 & 7.60 & 33.6 & 42.8 & 44.9 \\
\hline FC15 & 6.58 & 6.70 & 8.34 & 28.4 & 38.5 & 39.3 \\
\hline
\end{tabular}

L'analyse de ces résultats permet d'apporter les commentaires suivants :

- Les résistances de tous les mortiers augmentent régulièrement avec l'âge et ne présentent aucune chute ;

- La résistance à la compression diminue considérablement avec l'augmentation du pourcentage d'ajout à 7 jours ;

- Les résistances à la compression des mortiers pouzzolanique tendent à rejoindre celle du mortier témoin à 90 jours. 
La diminution de la résistance au jeune âge est attribuée principalement à l'activité lente de la PN. Ce phénomène s'explique par l'interaction entre la silice réactive qui se situe dans la partie vitreuse de la $\mathrm{PN}$ et le $\mathrm{Ca}(\mathrm{OH})_{2}$ libéré par l'hydratation du ciment CEM I ce qui donne à la PN la propriété de fixer la chaux. La réaction pouzzolanique n'est pas prédominante au jeune âge, ceci mène à une hydratation moins intense aux jeunes âges en induisant de faibles résistances (retardateur de prise) [8].

\subsection{Résistance chimique}

\subsubsection{Attaque par la solution de $5 \%$ d'acide sulfurique $\left(\mathrm{H}_{2} \mathrm{SO}_{4}\right)$}

La figure 5 montre la variation de la perte de masse, des éprouvettes confectionnées avec 0 , 15,20 et $25 \%$ de $\mathrm{PN}$, en fonction de la période d'immersion dans la solution de $5 \% \mathrm{H}_{2} \mathrm{SO}_{4}$. On constate une perte de masses permanentes concernant les mortiers et/ou composites immergés dans la solution d'acide sulfurique. D'après les résultats et après 56 jours d'immersion, nous constatons que l'ajout de 15, 20 et $25 \%$ de PN réduit la perte de masse, par rapport au mortier de référence, respectivement de 20, 17, $26 \%$.

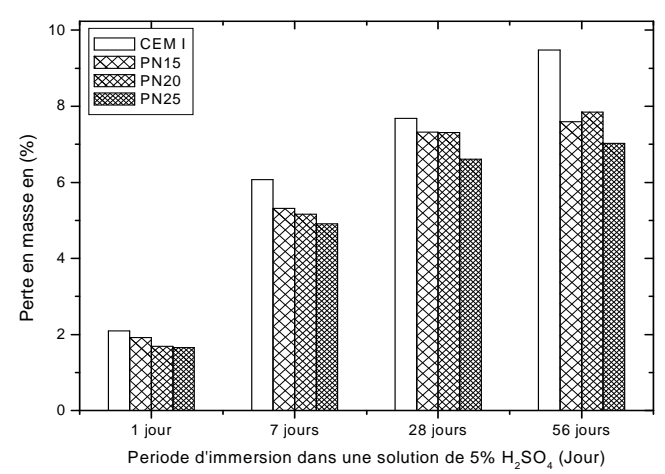

Fig.5. L'évolution de la perte du poids en fonction de la période d'immersion en $5 \% \mathrm{H}_{2} \mathrm{SO}_{4}$ (cas de $\mathrm{PN}$ )

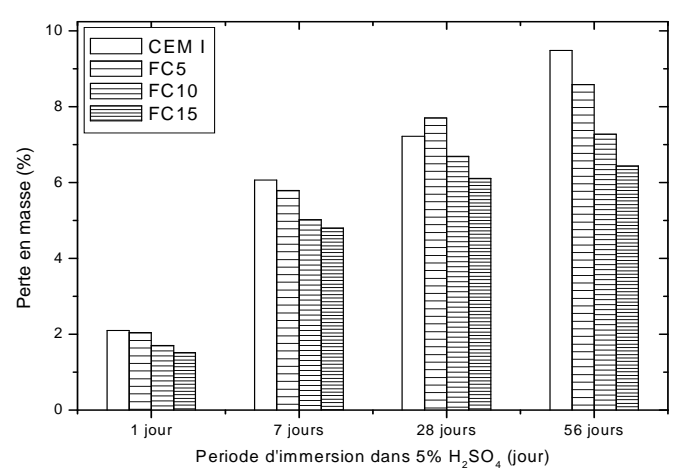

Fig.6. L'évolution de la perte du poids en fonction de la période d'immersion en $5 \% \mathrm{H}_{2} \mathrm{SO}_{4}$ (Cas des FC)

Comme pour l'ajout de la PN, la substitution du ciment par les FC augmente sa résistance chimique vis-à-vis de l'attaque de la solution de 5\% d'acide sulfurique. La figure 6 qui schématise le comportement des mortiers à base de $0,5,10$ et $15 \%$ de FC, montre qu'après 56 jours de conservation dans $5 \% \mathrm{H}_{2} \mathrm{SO}_{4}$ nous avons un gain de perte en poids de 10,23 et $32 \%$ pour les mortiers FC5, FC10 et FC15 respectivement par rapport au mortier sans ajout. 


\subsubsection{Attaque par la solution de $5 \%$ d'acide nitrique $\left(\mathrm{HNO}_{3}\right)$}

Dans le cas de l'attaque des mortiers par l'acide nitrique, nous remarquons d'après les figures 7, 8 que les deux ajouts minéraux (PN et FC) ont un apport bénéfique sur le ciment CEM I. La réduction de la perte en poids, après 56 jours d'attaque, des mortiers contenants 15,20 et $25 \%$ de PN est respectivement de 13, 22, 14.4\% par rapport au mortier de référence.

Le même constat pour le cas de l'ajout des FC (figure 8), l'incorporation de 5, 10, et 15\% de fillers améliore le comportement de ces mortiers en réduisant leur perte de poids de 18, 9.5, et $10.2 \%$ respectivement après 56 jours d'immersion dans la solution de $5 \% \mathrm{HNO}_{3}$.

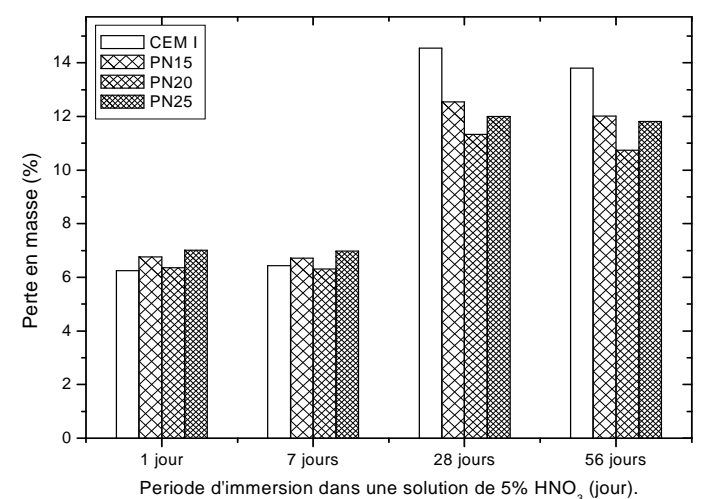

Fig.7. Evolution de la perte du poids en fonction de la période d'immersion en $5 \% \mathrm{HNO}_{3}$ (Cas de la $\left.\mathrm{PN}\right)$

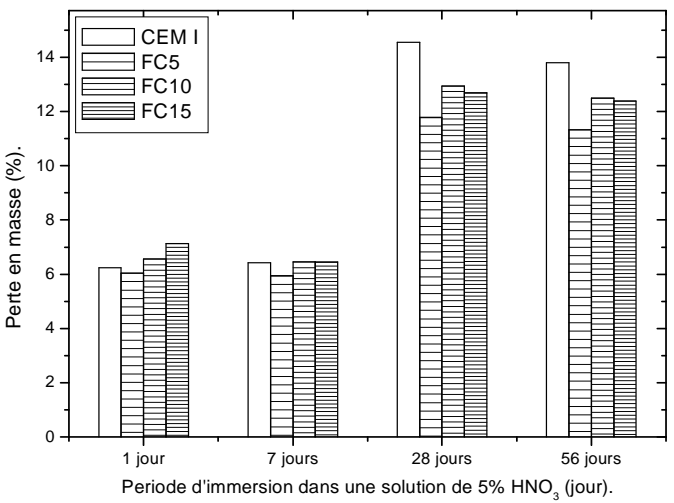

Fig.8. Evolution de la perte du poids en fonction de la période d'immersion en $5 \% \mathrm{HNO}_{3}(\mathrm{Cas}$ des FC)

La résistance chimique à l'attaque acide des mortiers contenant la PN peut être expliqué par l'amélioration de la liaison entre la pate et les agrégats suite à la conversion de la portlandite qui réagie avec la silice (composant principal de la $\mathrm{PN}$ ) pour former le gel de silicate de calcium hydraté (C-S-H) [8]. Pour le cas de l'ajout des FC, la bonne résistance chimique des mortiers à base de cet ajout minéral est due à la consommation de l'acide par les carbonate de calcium $\left(\mathrm{CaCO}_{3}\right)$, qui jouent le rôle de tampon.

Des conclusions similaires ont été faites par Safwan et Abou-Zeid [9]. Ils ont étudié la durabilité des bétons à base de fumée de silice avec un taux de remplacement de $40 \%$. Baltakys et al. [10] attribuent le rôle bénéfique de la substitution du ciment Portland par la pouzzolane naturelle ou bien la fumée de silice à plusieurs raisons : 
a) Le caractère pouzzolanique de ces matériaux réduit leur perméabilité, ce qui empêche la pénétration et le transport des ions agressifs;

b) Les matériaux pouzzolaniques améliorent les caractéristiques mécaniques et chimiques des matériaux cimentaires ainsi que leur résistance contre les fissurations et les expansions;

c) Le remplacement d'une partie de ciment par un matériau de faible alcalinité réduit le degré total des alcalins présents;

d) La réaction des pouzzolanes avec la chaux libre donne plus de silicate de calcium hydraté $(\mathrm{C}-\mathrm{S}-\mathrm{H})$ avec un rapport faible de $\mathrm{CaO} / \mathrm{SiO}_{2}$, ce qui mène à une décalcification progressive pour former d'autres hydrates tels que l'ettringite et le monosulfoaluminate, alors que la décalcification des $\mathrm{C}-\mathrm{S}-\mathrm{H}$, avec un $\mathrm{CaO} / \mathrm{SiO}_{2}$ élevé (cas des $\mathrm{C}-\mathrm{S}-\mathrm{H}$ issus de l'hydratation du ciment Portland), produit une structure vulnérable dans la solution d'acide sulfurique [10].

\section{INSPECTION VISUELLE}

La visualisation à l'aide du microscope optique a été utilisée pour observer la surface détériorée de spécimens à intervalles réguliers. Les Photographies des échantillons corrodés par l'acide sulfurique et l'acide nitrique sont montrés par les figures 9 et 10 respectivement, la détérioration de la surface a été observée à augmenter avec le temps si étendue de la détérioration dans les sept séries d'échantillons n'a pas pu être aisément différenciés par une inspection visuelle.

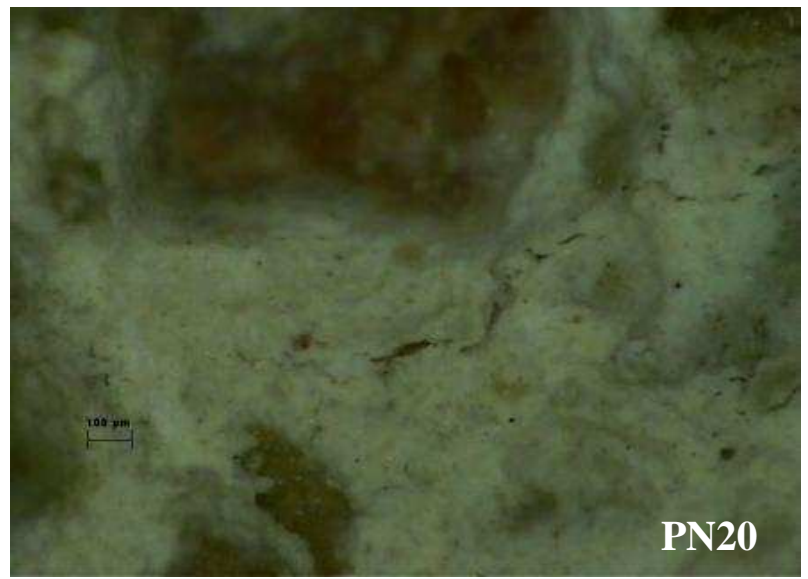

Fig.9. Etat de surface dans la solution de 5\% $\mathrm{H}_{2} \mathrm{SO}_{4}(\mathrm{X} 175)$

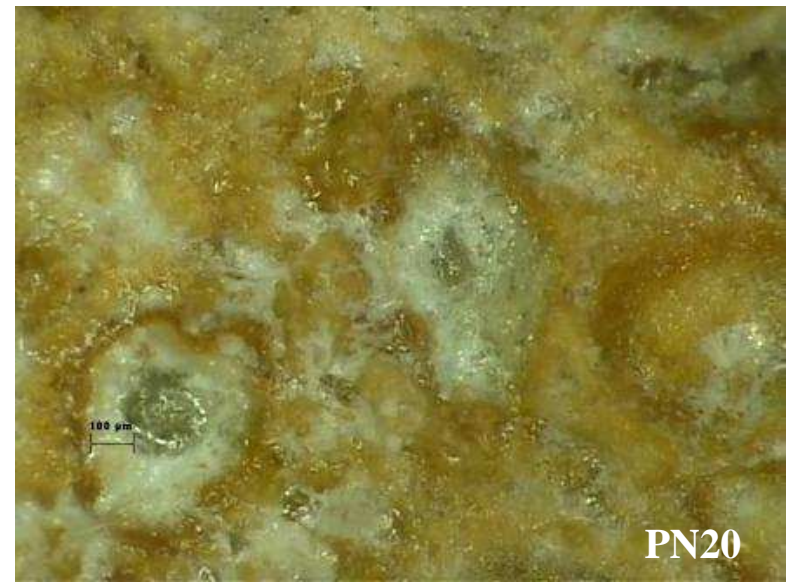

Fig.10. Etat de surface dans la solution de $\mathrm{HNO}_{3}(\mathrm{X} 175)$ 
La différence entre le taux de corrosion du mortier de control et les mortiers composés conservés dans les milieux acides est perceptible. Après 56 jours nous avons constaté que les mortiers à base de PN et des FC se sont mieux comportés que le mortier du ciment Portland.

\section{CONCLUSION}

Nos résultats ont permis de mettre en évidence, à partir des essais mécaniques et chimiques sur mortiers, couplés aux analyses par microscope optique, qu'il est possible d'obtenir des ciments composés en substituant une partie du ciment CEM I par des matériaux minéraux locaux finement broyé (PN et FC).

L'étude calorimétrique nous à permet de constater que le remplacement du ciment par la PN ou les FC diminue d'une façon significative la chaleur d'hydratation du ciment avec ajout, ce qui réduit l'effet du retrait de la pâte.

Dans le cadre de la revalorisation de ces deux ajouts minéraux (abondant en Algérie), tout en garantissant un gain économique certain, il est souhaitable de les utiliser comme ajout cimentaire à raison de $10 \%$ pour les $\mathrm{FC}$ ou $20 \%$ pour la $\mathrm{PN}$.

Sur le plan environnemental, quand on sait qu'une tonne de clinker dégage une tonne de $\mathrm{CO}_{2}$, il est évident que cette substitution réduirait l'émanation de gaz à effet de serre à raison dans les cimenteries, et partant, contribuera à la protection de la couche d'ozone.

\section{REFERENCES}

[1] Khatri R P, Sirivivatnanon V, Effect of different supplementary cementitious materials on mechanical properties of high performance concrete. Cem. \& Conc. Res., 1995, 25(1), 209-220.

[2] Mehta P K, Greening of the concrete industry for sustainable development, Concrete International, 2002, 24(7), 23-27.

[3] Chabi S, Mezghiche B, Guettala H, Etude de l'influence des additions minérales actives sur le comportement mécanique des ciments et mortiers, Courrier du Savoir, 2004, 05, 03-08.

[4] Gherdaoui C, Benmalek M I, L’influence des déchets industriels de carrière sur les performances des bétons, Seminaire SNGID07-ENSET Oran, 2007. 
[5] NF EN 196-9, Méthodes d'essais des ciments - Partie 9 : Chaleur d'hydratation - Méthode semi-adiabatique, 20 août 2004.

[6] NF EN 196-1, Méthodes d'essais des ciments - Partie 1: détermination des résistances mécaniques , Avril 2006.

[7] ASTM C267 "Chemical resistance of mortars, grouts, and monolithic surfacings and polymer concretes", Annual Book of American Society for Testing Materials, Philadelphia, vol. 4.05, 1997, pp. 130-135.

[8] Senhadji Y, Escadeillas G, Mouli M, Khelafi H, Benosman S, Influence of natural pozzolan, silica fume and limestone fine on strength, acid resistance and microstructure of mortar. J. Powder Technology, 2014, V254, 314-323.

[9] Baltakys K, Jauberthie R, Siauciunas R, Kaminskas R, Influence of modification of $\mathrm{SiO}_{2}$ on the formation of calcium silicate hydrate. Mater. Sci. Pol., 2007, 25(3), 663-670.

[10] Safwan A K, Abou-Zeid M N, Characteristics of silica-fume concrete. J. Mater. Civ. Eng. (ASCE), 1994, 6(3), 357-375.

\section{How to cite this article:}

Senhadji Y, Benosman A.S, Escadeillas G, Mouli M, Laoufi L and Khelafi H. Mortar incorporating supplementary cementitious materials: strength, isothermal calorimetry and acids attack. J. Fundam. Appl. Sci., 2016, 8(2), 232-243. 